\title{
UCRL-CONF-203584
}

LAWRENCE LIVERMORE NATIONAL LABORATORY

\section{Convex Crystal X-ray Spectrometer for Laser Plasma Experiments}

M. May, R. Heeter and J. Emig

\section{April 16, 2004}

15th Topical Conference on High-Temperature Plasma Diagnostics San Diego, California USA, 19-22 April 2004 
This document was prepared as an account of work sponsored by an agency of the United States Government. Neither the United States Government nor the University of California nor any of their employees, makes any warranty, express or implied, or assumes any legal liability or responsibility for the accuracy, completeness, or usefulness of any information, apparatus, product, or process disclosed, or represents that its use would not infringe privately owned rights. Reference herein to any specific commercial product, process, or service by trade name, trademark, manufacturer, or otherwise, does not necessarily constitute or imply its endorsement, recommendation, or favoring by the United States Government or the University of California. The views and opinions of authors expressed herein do not necessarily state or reflect those of the United States Government or the University of California, and shall not be used for advertising or product endorsement purposes. 


\section{Convex Crystal X-ray Spectrometer for Laser Plasma Experiments}

M. May, R. Heeter and J. Emig

Lawrence Livermore National Laboratory

Measuring time and space-resolved spectra is important for understanding Hohlraum and Halfraum plasmas. Experiments at the OMEGA laser have used the Nova TSPEC which was not optimized for the OMEGA diagnostic space envelope or for the needed spectroscopic coverage and resolution. An improved multipurpose spectrometer snout, the MSPEC, has been constructed and fielded on OMEGA. The MSPEC provides the maximal internal volume for mounting crystals without any beam interferences at either $2 \mathrm{x}$ or $3 \mathrm{x}$ magnification. The RAP crystal is in a convex mounting geometry bent to a 20 $\mathrm{cm}$ radius of curvature. The spectral resolution, $\mathrm{E} / \mathrm{dE}$, is about 200 at $2.5 \mathrm{keV}$. The spectral coverage is 2 to $4.5 \mathrm{keV}$. The MSPEC can record four separate spectra on the framing camera at time intervals of up to several ns. The spectrometer design and initial field-test performance will be presented and compared to that of the TSPEC. Work supported by U. S. DoE/UC LLNL contract W-7405-ENG-48 


\section{Introduction}

To diagnose indirect-drive inertially confined fusion (ICF) experiments, time and space-resolved X-ray spectra are needed. In these experiments, laser radiation heats the inside of a gold hohlraum or halfraum producing a plasma that emits intense X-rays. The $\mathrm{X}$-ray radiation drives the capsule implosion and influences the resulting fusion yield. Spectroscopy can provide crucial information on the plasma conditions $[1,2,3]$ such as radiation output, energy deposition rate, energy balance, etc. The measured spectrum can also be used to identify ionization species and determine both the charge state distribution (CSD) and the effective ionization state, $Z_{\text {eff }}[4,5]$. Estimates of the electron temperature from the CSD and $\mathrm{Z}_{\text {eff }}$ are possible in conjunction with either Thomson scattering measurements or atomic physics modeling.

A variety of X-ray spectrometers have been used successfully on laser produced plasma experiments at facilities such as NOVA [6] and OMEGA [7]. Each facility requires a different spectrometer design to accommodate the unique spatial constraints of the target chambers and to obtain the desired photon energy coverage, spectral resolution and temporal resolution for the experiment. The X-ray spectrometers are typically positioned in the harsh environment inside the target chamber as close as $10 \mathrm{~cm}$ from the target. The spectrometers must fit inside the diagnostic manipulator ports for OMEGA and NIF and must not interfere with the paths of the incident laser beams in the target chamber.

At OMEGA, our experiments require spectra between photon energies of 2 to 4.5 $\mathrm{keV}$. Our previous spectrometer was the TSPEC [6] which was originally designed for NOVA. It used a cylindrically bent convex crystal geometry and produced good spectra. However, the TSPEC spectral coverage was not adequate for our measurements. The hardware was not compatible with the OMEGA geometry and interfered with several of the incident laser beams. A new multipurpose spectrometer snout, the MSPEC, has been built and fielded on OMEGA for measuring spectra in the 2 to $4.5 \mathrm{keV}$ spectral range. The MSPEC is an improved design and solves many of the difficulties with the TSPEC. The MSPEC snout is compatible with the beam geometries, has a more useful spectral 
coverage than the TSPEC, and can have different magnification nose cones (e.g. 2x and $3 \mathrm{x})$. The spectrometer snout can accommodate multiple crystal geometries. This paper discusses the general concept of the MSPEC and the convex crystal geometry implementation, the MSPEC-C.

\section{MSPEC Spectrometer Design}

The MSPEC spectrometer snout is a new multipurpose design to measure spectra in the 2 to $4.5 \mathrm{keV}$ region. The conical snout can accommodate a variety of crystal configurations that include the convex (MSPEC-C) and the elliptical (MSPEC-E) geometries [8]. The MSPEC-C and MSPEC-E snouts are the same except for the crystal mounting. The MSPEC snout with a convex crystal mount and the $3 \mathrm{x}$ magnification nose cone is shown in Fig. \#1. The snout does not interfere with the laser drive beams and fits inside the any of the 6 ten inch manipulator (TIM) ports at OMEGA. The snout has four channels each consisting of a slit to direct light onto four parts of the same crystal or four different crystal mount geometries. With one crystal, the same spectrum at four different times can be measured. With four different crystal mounts, a broader spectral range can be covered during the same time interval of a laser pulse. The slits mount at the end of the nose cone. Filters are mounted both at the tip of nosecone and just in front of MCP; $25 \mu \mathrm{m}$ of Be in each location. The slits are $5-10 \mathrm{~mm}$ long in the dispersive direction and between $20-200 \mu \mathrm{m}$ in the spatial direction. The spatial imaging is done with a simple

pinhole camera concept. Several interchangeable nose cones allow different magnifications (presently $2 \mathrm{x}$ or $3 \mathrm{x}$ ) of the target. The MSPEC snout mounts to a standard 4 channel OMEGA framing camera positioned $38.1 \mathrm{~cm}$ from target chamber center (TCC). Each microchannel plate strip on the camera is $30-34 \mathrm{~mm}$ (spectral) by $6-7 \mathrm{~mm}$ (spatial) depending upon the specific camera. The MCP electrons are converted to visible light with a P-11 phosphor. The four spectrum are recorded on either visible film or a CCD camera.

The cylindrically bent convex crystal geometry is a very useful compact spectrometer design for laser produced plasmas. The details of this geometry have been published elsewhere [9]. The convex crystal geometry gives a large photon energy 
coverage at a modest spectral resolving power. The photon energy coverage is provided by the angular divergence of the entrance aperture and the convex curvature of the crystal. The divergence from the crystal gives the convex geometry a larger photon energy coverage than a flat crystal geometry. The convex geometry can be used to measure photon energies from 0.5 to $10 \mathrm{keV}$ with the appropriate choice of crystal.

For the Hohlraum experiments, the MSPEC-C was designed to record spectrum between 2 to $4.5 \mathrm{keV}$. The photon energy dispersion and spectral resolving power of the MSPEC-C was modeled with the geometrical formulas in reference 9 (see figure \#2). The choice of crystal was determined to be RAP with a $2 \mathrm{~d}$ of $26.121 \AA$ A bent with a radius of $200 \mathrm{~mm}$. The 3x magnification allows a $2 \mathrm{~mm}$ view of the target on the $6 \mathrm{~mm}$ high MCP strips. The entrance slits were $80 \mu \mathrm{m}$ wide. From geometrical ray tracing, we estimate the spatial resolution at the source to be $\sim 110 \mu \mathrm{m}$.

The resolving power $(\mathrm{E} / \Delta \mathrm{E})$ of the convex crystal geometry is a convolution of three effects, the crystal resolution, the effective MCP detector resolution and the source size (Fig. \#2). The inherent resolving power of RAP is greater than 1000 in this spectral range [10] and is larger than any other effect. The MCP detector resolution is about 50 $\mu \mathrm{m}$ which results in a spectral resolving power of 200 to 700 . The most significant contribution to the measured line widths is the effect of source size. The targets act as the slit of the spectrometer. Larger targets will produce a lower resolution spectrum. The convex crystal design cannot have an entrance slit in the dispersion direction. An entrance slit would mask part of the crystal reducing the spectral coverage. However, a better spectral resolution can be obtained by using a target-mounted aperture to limit the effective source size of large targets.

The targets for our experiments will be Halfraums or Hohlraums of scale 1/4 to 1 . The dimensions are between $400 \mu \mathrm{m}$ and $1600 \mu \mathrm{m}$. The resolving power assuming a 600 $\mu \mathrm{m}$ tall source is $\sim 100$ at $4.0 \mathrm{keV}$ and $\sim 250$ at $2 \mathrm{keV}$. For a $1600 \mu \mathrm{m}$ target, the resolving power is 40 to 90 , respectively. A better resolving power is obtained by the MSPEC-E configuration with this geometrical configuration.

\section{Sample Spectrum}


We present MSPEC-C spectra from experiments at OMEGA in February 2004. A $2 \mathrm{~mm}$ square gold foil was irradiated with $2774 \mathrm{~J}$ of energy from 6 beams. Two beams from each of Cone 1, 2 and 3 were used each having $\sim 462 \mathrm{~J}$ of energy. The laser pulse was nominally a $1 \mathrm{~ns}$ square beam in time. The resulting focused spot size was about 550 $\mu \mathrm{m}$ and resulted in a laser intensity of $1.7 \times 10^{15} \mathrm{~W} / \mathrm{cm}^{2}$. The MSPEC-C viewed the foil nearly edge-on or $79.2^{\circ}$ from the normal of the foil. Four spectrum were recorded with framing camera XRFC2 at OMEGA at approximately t $=530$ ps, 930 ps, 1390 ps and $1750 \mathrm{ps}$ from the time the laser beams turned on. The MCP high voltage is gated and propagates across each strip in $\sim 200$ ps. The reported times are the instant when the MCP pulse was at the center of the strip. The film image recorded at $930 \mathrm{~ns}$ is shown in Fig. \#3. The lasers heat the foil from below in the drawing. The emitting plasma is blown off of the foil in the downward direction. The photon energy is from left to right. The dark line just below the foil is emission that has saturated the detector.

Below the dark line, spectral lines can clearly be seen as the detector moves out of saturation. At these beam intensities the M-band lines of $\mathrm{Cu}$ to $\mathrm{V}$-like gold dominate the spectrum. There are several distinct line groups that can be seen the $4 p \rightarrow 3 d$ and $4 s \rightarrow 3 p$ below $2.4 \mathrm{keV}$, the $4 \mathrm{f} \rightarrow 3 \mathrm{~d}$ between $2.4-3 \mathrm{keV}$ and the $5 \mathrm{f} \rightarrow 3 \mathrm{~d}$ between $3-4 \mathrm{keV}$. These line groups have been previously identified in low density plasmas [11,12]. The MSPEC images were recorded on visible film that has a logarithmic response. The response of the film was calibrated out. Line-outs from the image were done. The $\mathrm{Cu}$ to $\mathrm{V}$-like $\mathrm{Au}$ $5 \mathrm{f} \rightarrow 3 \mathrm{~d}$ lines are shown in a sample line-out in Fig. \#4.

The dispersion curve was measured from these spectra and was very close to the predicted values. Therefore, the as-built configuration was close to the design. From the line widths one can estimate that the spectral line widths are $\sim 35 \mathrm{eV}$. The measured resolving power of the MSPEC-C at $3.5 \mathrm{keV}$ and $2.0 \mathrm{keV}$ is $\sim 100$ and $\sim 200$, respectively, and is consistent with the resolving power being limited by the source size.

\section{Acknowledgements}

This work was performed under the auspices of the U. S. Department of Energy by the University of California Lawrence Livermore National Laboratory under contract W7405-ENG-48. 


\section{Captions:}

Figure \#1: Schematic of the MSPEC-C spectrometer snout.

Figure \#2: Calculated spectral range and resolving power due to the crystal, the MCP detector and the source size and the measured spectral coverage.

Figure \#3: Film image recorded by the MPSEC-C of a Au disk at 930 ns from the start of the laser pulse.

Figure \#4: Measured calibrated spectrum from the MPSEC-C of the $5 \mathrm{f} \rightarrow 3 \mathrm{~d}$ emission lines from a $\mathrm{Au}$ Disk at $930 \mathrm{~ns}$ from the start of the laser pulse. 


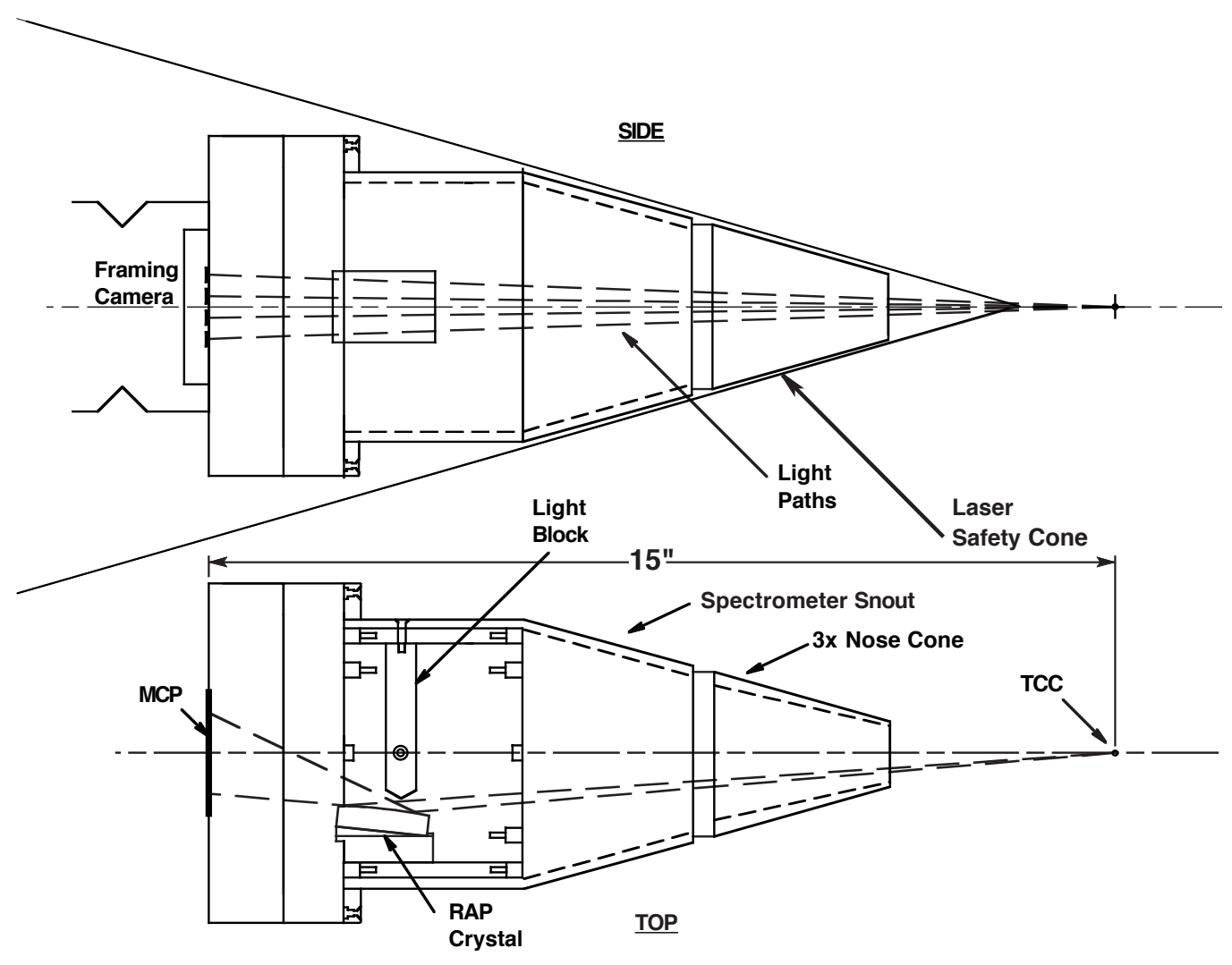

M.J. May: Figure \#1 


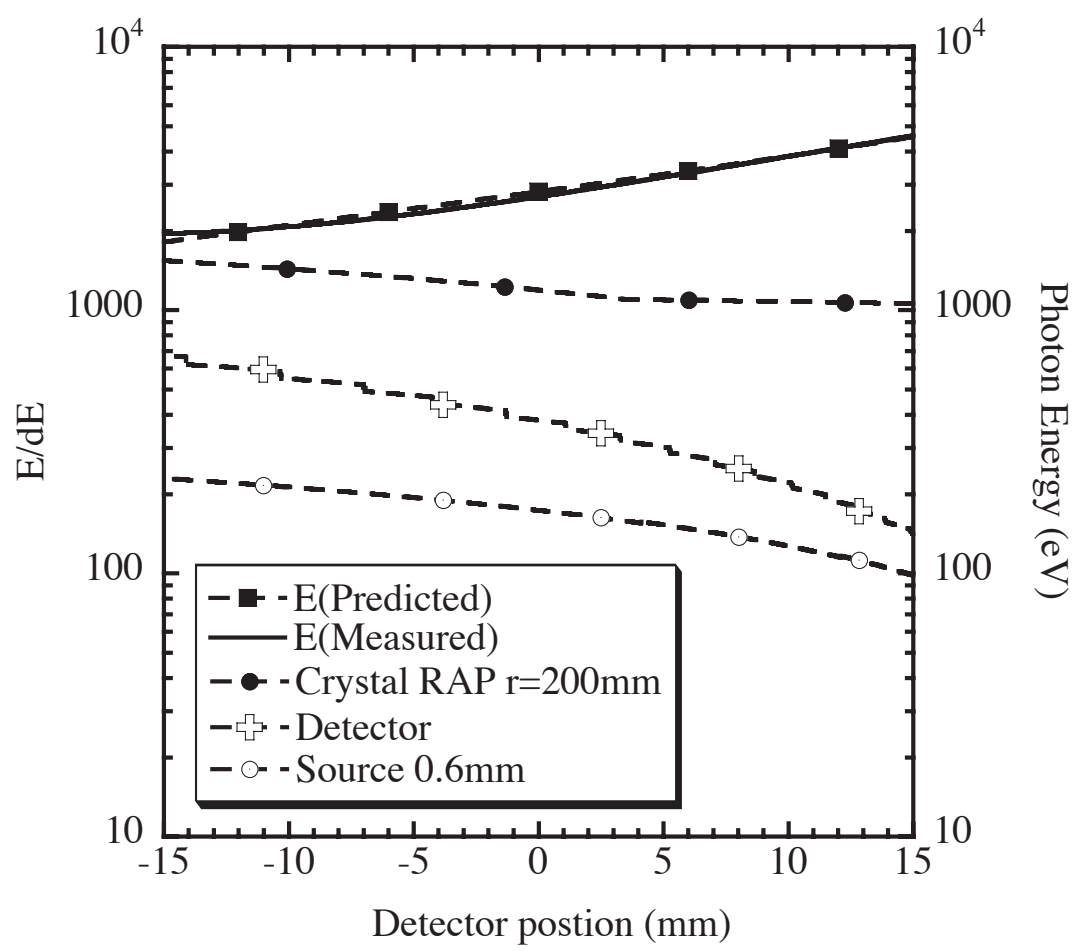

M.J. May: Figure \#2 


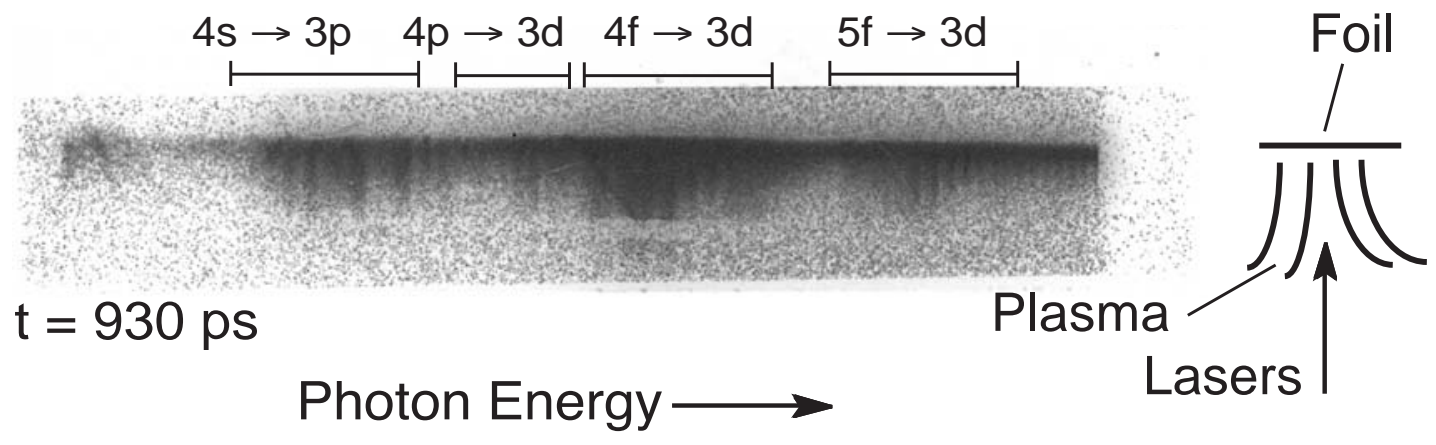

M.J. May: Figure \#3 


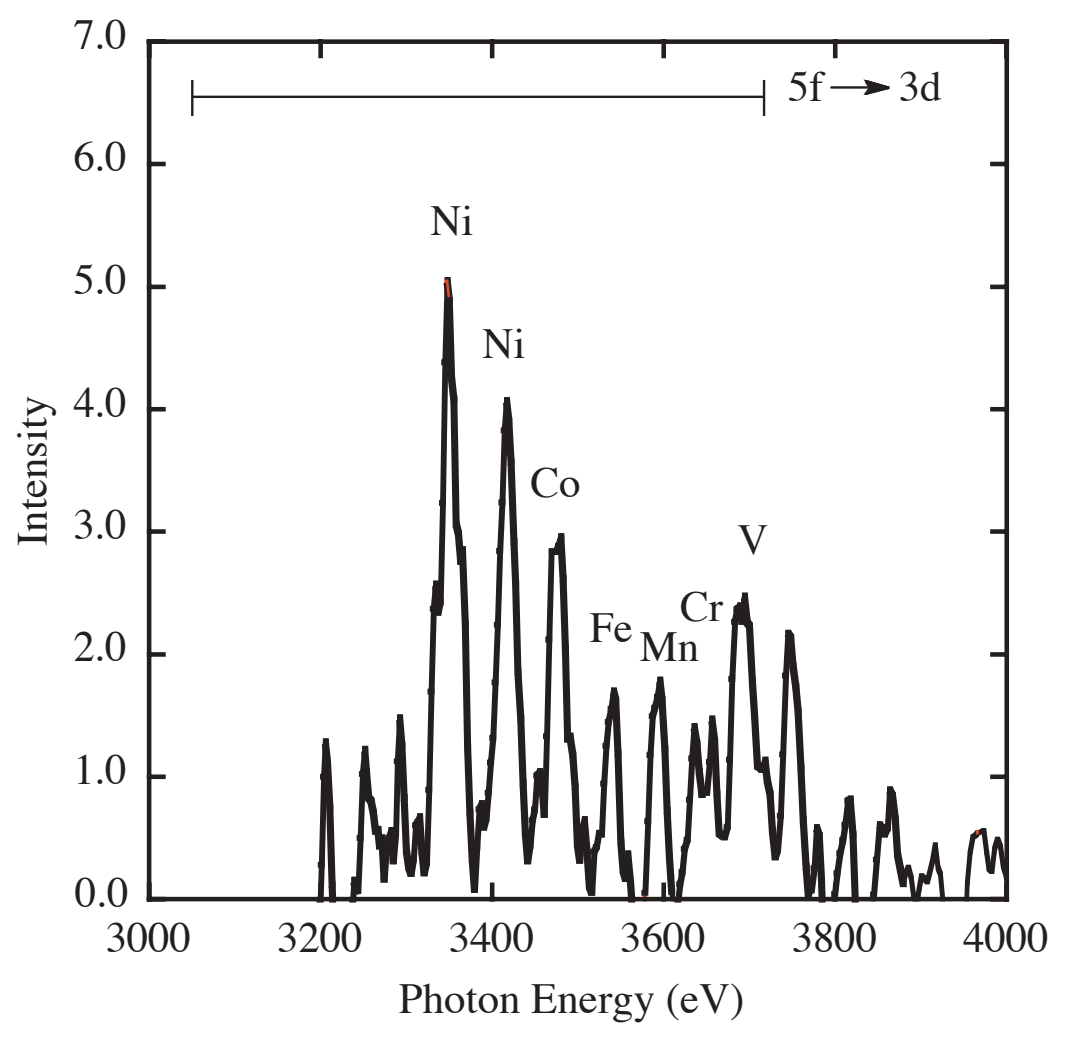

M.J. May: Figure\#4 


\section{References:}

${ }^{1}$ R. L. Kauffman, in Handbook of Plasma Physics, Vol. 3, edited by Rubenchik and Witkowski (Elsevier Science, North-Holland, Amsterdam, 1991), pp. 111-162 and references therein.

${ }^{2}$ T. S. Perry, P. T. Springer, D. F. Fields, D. R. Bach, F. J. D. Serduke, C. A. Iglesias, F. J. Rogers, J. K. Nash, M. H. Chen, B. G. Wilson, W. H. Goldstein, B. Rozsynai, R. A. Ward, J. D. Kilkenny, R. Doyas, L. B. Da Silva, C. A. Back, and R. Cauble, Phys. Rev. E, 54, 5617 (1996).

${ }^{3}$ C. A. Back, R. L. Kauffman, P. M. Bell, and J. D. Kilkenny, Phys. Rev. Lett., 77, 4350 (1996).

${ }^{4}$ S. H. Glenzer, K. B. Fournier, B. G. Wilson, R. W. Lee, and L. J. Suter, Phys. Rev. Lett., 87, 045002 (2001).

${ }^{5}$ M. E. Foord, S.H. Glenzer, R.S. Thoe, K.L. Wong, K.B. Fournier, B.G. Wilson, and P.T. Springer, Phys. Rev. Lett., 85, p 992 (2000).

${ }^{6}$ C.A. Back, S.H. Glenzer, O.L. Landen, B.J. MacGowan and T.D. Shepard, Rev. Sci. Instrum., 68, p 831 (1997).

${ }^{7}$ J. Seely, C. Back, R, Deslattes, L. Hudson, G. Holland, P. Belland, M. Miller, Rev. Sci. Instrum., 72 No. 6, p 2462 (June 2001).

${ }^{8}$ R. Heeter, J. Emig, M. May and B. Young, Review of Scientific Instruments, this issue.

${ }^{9}$ L. Koppel and J. Eckels, Lawrence Livermore National Laboratory Report, UCRL79781 (1977).

${ }^{10}$ J. Burek, Space Instrumentation, 2, p 53-104 (1976).

${ }^{11}$ M.J. May, K.B. Fournier, P. Beiersdorfer, H. Chen, and K.L. Wong, Phys. Rev. E, 68, p 036402 (2003).

${ }^{12}$ K.L. Wong, M.J. May, P. Beiersdorfer, K.B. Fournier, G. V. Brown, S. B. Utter and P. Springer, Phys. Rev. Lett. 90, No. 23, p 235001-1 (13 June 2003). 